\title{
Near-Death Experiences in India: Prevalence and New Features
}

\author{
Satwant K. Pasricha, Ph.D. \\ National Institute of Mental Health and \\ Neurosciences, Bangalore, India
}

\begin{abstract}
$A B S T R A C T$ : In order to learn about the prevalence rate in a larger Indian population and to explore new features of near-death experiences (NDEs), I systematically surveyed 60 villages in southern India covering a population of 36,100 persons. The prevalence rate was 4 cases per 10,000 persons. Unlike the Western studies that have been generally conducted in hospital settings, the present study was conducted in the community setting. I systematically studied new features of visibility in the other realms, the experience of bright but soothing light, sensation of a cave, extrasensory experiences, and attitude toward life and death. In spite of different locations (community versus hospital) of the experiencers at the time of NDEs in the Indian and Western cases, common features across cultures suggest the presence of some universality of features, lending support to my earlier thesis that commonalty in features is indicative of a genuine phenomenon underlying NDEs.
\end{abstract}

KEY WORDS: near-death experience; India; culture; crosscultural variation.

I had previously, with Ian Stevenson, published a preliminary report of 16 cases of near-death experiences (NDEs) that we investigated in north India, comparing their features with the features of 78 American NDEs (Pasricha and Stevenson, 1986). However, we did not employ any systematic methods of sampling for selection of the cases for that study. Subsequently, I conducted systematic surveys in

Satwant K. Pasricha, Ph.D., is Professor of Clinical Psychology at the National Institute of Mental Health and Neurosciences (NIMHANS). The author thanks NIMHANS for support of this research. Sreekante Gowda provided assistance in data collection. Bruce Greyson and Emily Williams Kelly offered helpful suggestions for the improvement of this paper. Reprint requests should be addressed to Dr. Pasricha at the National Institute of Mental Health and Neurosciences (NIMHANS), Bangalore 560 029, India; e-mail: drpasricha@gmail.com. 
the Channapatna region of Bangalore, the capital city of Karnataka in southern India, to learn about the prevalence rate of NDE cases. I published the findings in two separate reports as the surveys, although part of the same project, were conducted at two different points in time (Pasricha, 1993, 1995). The prevalence rate ranged from 1 to 2 cases per thousand people. It was not clear whether the variation in prevalence rate was a true variation or due to difference in the size of the populations sampled.

When I compared features of cases from northern and southern India, I found some of them to be common to both regions and seemed typical of Indian cases, but I also observed variations among other features. Some of the varied features of the Indian cases were similar to the ones reported in American cases. The typical features of Indian cases generally included experiencers going to other realms with Yamdoots (Yamatoots) or messengers of the god of death, being passed on to a man with a book, discovering that they were mistakenly brought there instead of another person or their time had not yet come to die and they were to be sent back, or sometimes meeting deceased relatives who asked them to go back to terrestrial life. The experiencers usually returned to terrestrial life with the same messengers or deceased relatives or came back alone.

\section{Method}

\section{Source of Data}

In 1992 and 1994, I conducted another survey in a region of the Bangalore district, the findings of which are presented in the present article. The aims of the survey were to determine the prevalence rate of NDEs in a larger Indian population, to learn about new features not reported in the earlier series of cases in India, and to learn about the influence of such NDEs on the experiencers.

For this purpose I chose Hoskote, a taluk (roughly corresponding to a county in the United States and the United Kingdom) in the Bangalore district, situated on the Bangalore-Madras (Chennai) highway about 30 kilometers northeast of Bangalore. The reasons for selecting this particular region were that it represented both rural and urban populations, and was easily accessible from the National Institute of Mental Health and Neurosciences, where I work. The Hoskote taluk consisted of 362 villages, of which 52 had been listed as 
uninhabited (Director of Census Operations, 1984). Out of the remaining 310 inhabited villages, 60 villages with a total population of 36,100 persons were selected, by using computer generated random numbers.

The principal method employed for data collection was interviews, which I conducted in two stages.

\section{First Stage Interviews}

During the first stage, I interviewed the principal respondents to learn about their knowledge of cases. For the purpose of selecting the principal or target respondents, I used voter registration lists and chose one member from every household, usually the head of the family. Thus I identified a total of 6,017 persons from the 60 villages, of which 5,809 could be contacted for the purpose of interviews, the remaining respondents being unavailable on two subsequent calls. To gain the confidence and cooperation of the villagers, I enlisted the assistance of the schoolteachers and /or village leaders.

\section{Second Stage Interviews}

During the second stage I interviewed a total of 16 near-death experiencers who were identified by the principal respondents. The inclusion criteria for including cases in the prevalence count were that the experiencers had to be alive at the time of the survey and they had to be residing in the survey villages at the time.

I interviewed the identified experiencers for a detailed account of their NDEs. I also interviewed their relatives, when they were available and had reliable information, to learn about the physical condition and other circumstances concerning the experiencers' NDE and to corroborate any information regarding the NDE that the experiencer had shared with them. I first allowed the experiencers to describe their NDEs spontaneously, and later filled in any missing information by questioning them with the help of a checklist prepared for the purpose, which included all 12 features that were reported in the previous studies (Pasricha, 1993, 1995; Pasricha and Stevenson, 1986).

As we proceeded with the investigations, the experiencers spontaneously reported some new features that seemed important, and I added them to the checklist. However, I was not able to ask all 
experiencers about all features from the checklist, either due to lack of time or their level of comfort with the questions concerning details of features. The interviews lasted for about 45 minutes to an hour or more.

\section{Investigation of Additional Cases}

In the previous studies, variations in the features were reported in cases selected from northern and southern India, as well as among cases selected from the same general region of southern India. In view of these variations, I decided to increase the sample size in order to cover a larger number of features, including any new ones. I therefore interviewed additional experiencers who were reported by the principal respondents as cases in the first stage interviews, but who currently lived outside the identified survey villages. Keeping in view the stipulated time for completion of the project and available resources, I identified 55 such experiencers who lived within 20 kilometers of Hoskote. Of those 55 experiencers, 18 had died, 9 had been incorrectly identified as experiencers, and 6 had moved to other villages. I investigated the remaining 22 cases from outside the survey villages and added them to the 16 cases investigated from within the survey villages. Thus the final analysis was carried out on 38 cases. As I mentioned above, the number of experiencers varied for different features because I was not able to ask details of all questions to all the experiencers, and I added some of the questions later, during the course of the study.

In addition to interviewing the experiencers themselves, I was able to interview 10 of their relatives.

\section{Results}

\section{Prevalence of NDEs}

Occurrence of cases varied across villages. Three cases each were reported from two villages, two cases from one village, one each from 13 villages, and no cases were reported from 44 villages. In all, 21 cases were reported from 60 villages with an estimated population of 36,100 ; one of these did not meet the inclusion criteria, hence was not included in the final count. Thus the prevalence rate came to about 0.4 case per thousand persons or 4 cases per 10,000 persons. 
Of the 20 cases reported from the survey villages, the experiencers in two cases had moved out of their respective villages when we returned for investigating their cases, and a third was not available for further interviews. Thus 17 experiencers were investigated. Of those I excluded from the analysis one case for which details other than the demographic features were not available. I analyzed the remaining 16 cases from the survey villages and the additional 22 cases from outside the survey villages.

\section{Physical Health and Location of Experiencers at the Time of the NDE}

Data on physical condition of the experiencers at the time of the NDE were available in 34 cases. Ten of the experiencers were not suffering from any illness up till the time of NDE; two women in this group had NDEs during childbirth. The remaining 24 experiencers had various physical problems at the time of NDE, including fever ( 8 cases), plague (7cases), smallpox ( 3 cases), wounds ( 3 cases), tuberculosis, general weakness, and jaundice (one case each). All but two experiencers had their NDEs at home; two experiencers reported having NDEs while in the hospital. One of them had the NDE during a surgical operation and the other one had it a few days prior to his operation.

\section{Case Reports}

Before providing the details of the features, I will acquaint readers with some accounts of the Indian cases. In order to do that, I shall next present three brief case reports.

The Case of Munnichinnappa. We interviewed the experiencer in this case, Munnichinnappa, in February 1995. He had been born in 1946. According to his wife, Parvathamma, sometime in 1958 around 10:30 A.M. he fell from a tree and "died." All his relatives were informed about his death. About 3:00 P.M. he came back to life and told his family how he was taken to Yamaloka and sent back.

Yama, the god of death, is referred variously as Yamraj by northern Indians and Yamaraja by southern Indians; his messengers as Yamdoots; and the place where he as Yamapatna or Yamaloka. A number of references to Yamraj, Yamdoots, and Chitragupta ("the man with the book") can be found in the Hindu scriptural literature (Hazra, 1940/1975; Walker, 1968). 


\section{Munnichinnappa narrated his experience as follows:}

Two big black persons with big mustaches came and asked me to go along with them. When I resisted, they held my hand and took me forcibly. While going, I saw a big forest with big trees and it was dark there. We walked some distance and came across a big river. After crossing the river, we reached a place where the light was very bright, brighter than the sunlight, and people there looked very strange. When I went inside that place, I saw Yamaraja was sitting in a chair with many other persons. I was taken to one person who was sitting in a corner with a big book. He looked at me and said, "I did not ask for this person. Send him back; I had asked for a different person." I did not know how to get back; the two persons who had taken me there came with me and left me outside the village.

The Case of Annamma. We interviewed Annamma in June 1995 when she was 75 years old. At about the age of 40 , she had a quarrel with her husband, during which he hit her on the head with a stick. As a result she fell down and lost consciousness instantly. No other family member was available to provide details about the severity of Annamma's apparent head injury or the duration of her unconscious state. She said that she was "unconscious" for about 2 hours and had the following experience during that time:

One lady came and asked me to follow her, which I did. We walked through a forest; there were big trees. After some time we reached a big place that looked like a city. There were many people and they were busy with their work. That lady took me into a big house and left me at the gate to go inside. When I went in, I saw Yamaraja; a huge black person was [also] sitting there. On that person's questioning I told him my name and he said, "You don't have a seat here; you go back." Yamaraja also told me to go back and asked a person to take me out. One black person came and took me to a place where fire was burning. He burned me with a burning wood on the right leg (below the knee) and called the same lady (who had brought me there) to take me back. While coming from there, I saw my father there (who had died six years earlier). He inquired about the people of the family and asked me to go back. We followed the same route and came back. When I reached the village, I saw my body was lying on a mat.

The Case of Srinivasa Reddy. We interviewed Srinivasa Reddy in July 1995 when he was 75 years old. He had had the experience around the age of 35 . He was suffering from fever for about a week and was taking medication. His wife, Muniyamma, was away at her parents' home where she had gone for the delivery of their second child. When she was informed that her husband was not well, she 
returned back home in the evening. On reaching home, she found him unconscious and talking irrelevantly for some time, and then he completely stopped talking. She and others around noticed that his breathing had stopped, so they thought he was dead and sent for his relatives. Since it happened in the evening, they laid the body outside in the open, as is customary, and waited for the relatives to arrive. Suddenly about 8:00 or 9:00 P.M., he shook his head and legs and started breathing; they all felt very happy to have him back. The next morning he narrated his experience to them.

Srinivasa Reddy gave us the following account of his experience:

Two people came and asked me to go with them. I went with them walking on a mud road. We walked for a long time and reached a big place, which was like a city. There were big houses and many people. We went inside a big house where I saw a man with a big book to the left of Yamraj. The man with the book said, "Why have you come here?" I said, "These people have brought me here." He said, "You are not required here; I had asked for Ashwath Reddy. You go back," and asked those two persons to bring Ashwath Reddy. We came out; while coming out I told them that I was suffering from fever; could they give me some medicine to cure my illness? They took me to an old lady who gave me some herbal juice which I drank and came back with them; they left me outside my village.

After I came back [from the other realm] my fever came under control quickly and since then I have not suffered from fever even once. For this reason the memory of my experience is still fresh in my mind.

Muniyamma told us that one Ashwath Reddy in their village, who was about 80 years old and was ill for sometime, died that day. Her husband apparently did not know of his death, although it is possible that he knew of his illness. However, I could not independently learn about Ashwath Reddy's death.

\section{Features of the NDE Cases}

I shall next describe demographics and present the main features of these NDE cases in tabular form, along with the features reported in the earlier series, followed by the new features detected during the present study.

Demographic features of the cases. Twenty-one (55 percent) of the experiencers were male and 16 were female. The median age of the experiencers at first interview was 72 years, with a range from 30 to 108 years; their median age at the time of the experience was 36 years, 
Table 1

Main features of the cases

\begin{tabular}{lr}
\hline \multicolumn{1}{c}{ Feature } & Number (percent) of experiencers* \\
\hline Visited other realms & 35 of $37(95 \%)$ \\
Went with messengers or relatives & 33 of $35(94 \%)$ \\
Went alone & 2 of $35(6 \%)$ \\
Met deceased relatives or acquaintances & 9 of $27(33 \%)$ \\
Passed to a man with a book & 26 of $35(74 \%)$ \\
Met a being of light or religious person & 14 of $27(52 \%)$ \\
Sent back because of a mistake & 33 of $37(89 \%)$ \\
Sent back by others for other reason & 2 of $37(5 \%)$ \\
Came back by own free will & 2 of $37(5 \%)$ \\
Came back with messengers or relatives & 23 of $35(66 \%)$ \\
Another person died instead of experiencer & 12 of $35(34 \%)$ \\
Life review & 0 of $13(0 \%)$ \\
Out-of-body experience & 5 of $27(19 \%)$ \\
Residual marks from having been branded & 15 of $32(47 \%)$ \\
Cave & 1 of $25(4 \%)$ \\
\hline
\end{tabular}

* number of experiencers reporting each feature out of number of experiencers for whom information was available varied for different features.

with a range from 5 to 103 years; and the median interval between the experience and the interview was 21 years, with a range from 2 to 70 years. Information about experiencers' occupations was available for 36 cases, of whom 18 (50 percent) were farmers, 8 (22 percent) were housewives; 2 (6 percent) each were businessmen, laborers, and astrologers; 1 ( 3 percent) was a social worker, and 5 ( 6 percent) were retired.

Main features of the cases. Table 1 shows the main features of the cases, with the number of experiencers reporting each case and the percent of experiencers for whom information was available regarding that feature reporting each feature. Most of the features are self explanatory but a few require more elaboration. A majority of the experiencers reported having gone to the other realms with the messengers of the god of death or with deceased relatives. Nearly three-fourths of them reported having been passed to the man with a book, who discovered that they had been taken there by mistake in place of another person or they were not yet scheduled to die, resulting in their being sent back to terrestrial life either by the same 
messengers or alone. Fifteen (47 percent) of the experiencers reported having been branded in the other realm, and had residual marks upon regaining consciousness. All these features are typical of Indian cases and were reported by most of them.

In addition, some of the experiencers reported features that have also been reported from other cultures, mainly from the United States and the United Kingdom. These included out-of-body experiences (5 cases) and revival through one's own will ( 2 cases) or being sent back to terrestrial life by others ( 2 cases). However, as in my previous surveys of Indian cases, no experiencer reported a life review or panoramic memory, or passing through a tunnel.

\section{New Features of NDE Cases}

In this category I have included features that either were reported for the first time among Indian cases by the experiencers in this study or had not been systematically investigated in previous Indian studies. Table 2 shows new features that were added to the checklist at a later stage of investigation. In cases where the experiencers did not mention them spontaneously, I specifically asked about these features.

Visibility and intensity of light in the other realms. Twenty-seven experiencers answered the question about visibility in the other realm. A little over half of them ( 52 percent) reported the other realm to be normal to very bright (41 percent), so that they could see the things clearly. A small number of experiencers ( 7 percent) said that it was dark there and the visibility was poor.

Twenty-one experiencers answered the question about the intensity of light. A little over half of them (52 percent) reported having seen bright light in the other realm. Their descriptions included "The light was so bright there that I could not open my eyes for some time" and "There was a bright light; I had never seen such a light before." One of them said that although the light was bright, it was cool or soothing.

Experience of a cave. I asked 25 experiencers a question about having a sensation of a tunnel. In response, one female experiencer reported having had an experience of a "cave." She reported: "[While coming back from the other realm] I was shown a road to go back which was a narrow road; it was like a cave."

Attitude toward life and death. In my previous surveys of Indian cases, I did not ask systematically about change in attitude toward 


\section{Table 2}

New features of cases

Feature

Number (percent) of experiencers*

Attitude toward death

Lost fear of death

Developed fear of death

No change in fear of death

19 of $34(56 \%)$

0 of $34(0 \%)$

15 of $34(44 \%)$

Attitude toward life

Became more religious and generous

14 of $34(41 \%)$

Visibility on other realms

Normal visibility

14 of $27(52 \%)$

Brighter than normal

11 of $27(41 \%)$

Darker than normal

2 of $27(7 \%)$

Intensity of light

Bright

11 of $21(52 \%)$

Experience of a cave

1 of $25(4 \%)$

Extrasensory experiences

* number of experiencers reporting each feature out of number of experiencers for whom information was available varied for different features.

death following the NDE. Experiencers in a few cases spontaneously mentioned about their attitude toward death. However, I did not specifically record their attitude toward life in any of the previous surveys. In the present study, I did inquire systematically about the experiencers' attitudes toward both life and death; therefore I have included these features together under the heading of new features.

Thirty-four experiencers answered the question concerning attitude toward death. More than half of them ( 56 percent) reported having lost fear of death, while the remaining 44 percent reported no change, but said they were not afraid of death prior to or following the experience.

Thirty-four experiencers answered the question regarding change in attitude toward life following the NDE. More than one-third of them (41 percent) reported that their religiosity and generosity had increased following the experience.

Extrasensory experiences. Twenty-three experiencers answered the question about extrasensory experiences following the NDE. Four of 


\section{Table 3}

Features of cases in all surveys from India with personal interviews

\begin{tabular}{|c|c|c|c|c|}
\hline Feature & $\begin{array}{c}\text { Pasricha and } \\
\text { Stevenson (1986) }\end{array}$ & $\begin{array}{c}\text { Pasricha } \\
(1993)\end{array}$ & $\begin{array}{c}\text { Pasricha } \\
(1995)\end{array}$ & $\begin{array}{c}\text { Present } \\
\text { study }\end{array}$ \\
\hline Saw own physical body & $10 \%$ & $14 \%$ & $0 \%$ & $19 \%$ \\
\hline $\begin{array}{l}\text { Taken to other realm by } \\
\text { messengers }\end{array}$ & $75 \%$ & $85 \%$ & $71 \%$ & $92 \%$ \\
\hline Saw deceased acquaintances & $25 \%$ & $58 \%$ & $25 \%$ & $33 \%$ \\
\hline $\begin{array}{l}\text { Saw beings of light or } \\
\text { religious figures }\end{array}$ & $75 \%$ & $0 \%$ & NA* & $52 \%$ \\
\hline Passed to a man with a book & $50 \%$ & $83 \%$ & $70 \%$ & $70 \%$ \\
\hline $\begin{array}{l}\text { Sent back because of mistake/ } \\
\text { not yet time }\end{array}$ & $62 \%$ & $60 \%$ & $46 \%$ & $89 \%$ \\
\hline Life review & NA & NA & NA & $0 \%$ \\
\hline $\begin{array}{l}\text { Other person to die in place of } \\
\text { experiencer }\end{array}$ & $44 \%$ & $30 \%$ & $11 \%$ & $34 \%$ \\
\hline Sent back for other reason & $0 \%$ & $10 \%$ & $11 \%$ & $5 \%$ \\
\hline Returned by own volition & $6 \%$ & $0 \%$ & $18 \%$ & $5 \%$ \\
\hline $\begin{array}{l}\text { Brought back by messenger/ } \\
\text { deceased guide }\end{array}$ & $81 \%$ & $88 \%$ & $56 \%$ & $66 \%$ \\
\hline Residual marks on body & $25 \%$ & $33 \%$ & $33 \%$ & $47 \%$ \\
\hline \multicolumn{5}{|l|}{ Change in attitude toward death } \\
\hline Lost fear & NA & NA & $20 \%$ & $44 \%$ \\
\hline Developed fear & NA & NA & $10 \%$ & $0 \%$ \\
\hline No change & NA & NA & $70 \%$ & $41 \%$ \\
\hline
\end{tabular}

*information not available.

them (17 percent) reported unexpected and quick recovery in their health following the experience, implying occurrence of apparent paranormal healing or accelerated healing processes; three (13 percent) were credited with the paranormal knowledge of another person's impending death; and two (9 percent) reported having out-ofbody experiences.

\section{Comparative Features of Indian Cases}

Table 3 highlights a comparative summary of features of the Indian cases investigated in different points in time. I have not included the 
study by Susan Blackmore (1993) because her main focus of study was on the feature of tunnel sensations. Moreover, she identified experiencers through a newspaper advertisement and did not conduct her interviews with the experiencers personally, but rather corresponded with them through the mail. Her study also had other methodological and interpretative flaws (Kellehear, Pasricha, Stevenson, and Cook, 1994).

All of the studies included in Table 3 employed personal interviews for eliciting details of NDEs from the experiencers as well as the concerned informants. Three of these studies identified cases through systematic surveys.

\section{Discussion}

In $44(73 \%)$ of the 60 villages surveyed, no case of NDE was reported. The estimated prevalence rate of NDE cases was 1 in 2500 or 4 cases in 10,000 persons, which is far below the rate reported in the earlier Indian studies (Pasricha, 1993, 1995). This indicates that the cases are not uniformly distributed across villages even within the same general area. It was observed in the previous two studies that with increase in the sample size, the number of NDE cases dropped; such a change in prevalence rate is known to occur but may not be the only factor. The low prevalence rate in the present study could have been due to one or all of the following: (a) larger population samples; (b) underreporting of cases; and (c) true variation in occurrence of cases. Variations in prevalence rates have also been reported in the Western studies. I am aware that the methods I used for identification of NDE cases are different from the ones used by Western researchers and hence cannot perhaps be compared. I have used a two-stage interview method for estimating the prevalence rates which none of the Western researchers seem to have used and hence the prevalence rates are likely to be different. However, we still need to look for an explanation to understand why variations in prevalence rates occur even when the studies are conducted by the same investigator using more or less same methodologies, and why similarities in the features have occurred in spite of different methodological approaches. It is reasonable therefore, to suppose, at this stage, that methodological variations alone cannot account for the differences in prevalence rates. 
As I mentioned in the section on methods, all questions related to the items of the checklist were not fully answered by the experiencers, due to either lack of time or the experiencer's level of comfort with the questions. This is a methodological problem inherent in community based or field studies; a certain amount of bias can not be eliminated. However, in the present study the experiencers generally responded to the main items of the checklist, but the details of their responses varied. For example, some experiencers gave a detailed account of the other realm or Yama, while others did not say more than that they met him or they were summoned by him, or responded to the question in the affirmative or in the negative. Hence variations in their reporting would not have influenced the data much since most of them responded to the main items.

We saw differences in features across two cultures when we compared features of Indian and American NDE cases in our first study (Pasricha and Stevenson, 1986). However, in the subsequent studies, differences in features were reported even in the cases investigated in northern and southern India, two parts of the same country (Pasricha, 1993). Furthermore, features not reported in one series of cases were reported in another series of cases in the same region. For example, out-of-body experiences, a frequent feature of Western cases, were reported in one of the two Indian series

The samples in the Western studies have been drawn mostly from the hospital populations whereas samples for the present and earlier Indian studies were drawn from the community populations. Unlike the hospital based samples, it is generally not possible to determine the exact degree of closeness to death and the time lapse between apparent death and the experience in the community based samples on which the present and earlier studies were carried out in India. Although relatives and others around the experiencers were able to establish reasonably that a person had "died" (in some cases they even performed the precremation rituals) or appeared to be dead to them, their estimates of the duration of apparent death vary considerably (Stevenson, Pasricha, and McClean-Rice, 1989). It is also difficult to get accurate estimates of time lapse between death and the experience. In spite of these difficulties, it is reasonable to assume that the person was close enough to death when he or she had the experience that resembled an NDE where death and gap between the death and experience could be established more precisely.

In spite of the larger sample size and newer features coming up in the present study, the features of a life review or panoramic memory 
and of a tunnel were not reported in any of the Indian series, including the present one. As I conjectured in a previous study (Pasricha, 1995), the first of these features, the life review, does occur in Indian cases but perhaps through a different process. In the Western cases, experiencers often go through their own life review by themselves, whereas in the Indian cases the "man with a book" in the other realm may review the experiencer's life. Blackmore (1993) did report the feature of a tunnel in Indian cases in a study. However, as mentioned earlier, her experiencers were self selected from a large city and were apparently literate, as they responded to an English language newspaper advertisement; some of them even might have been familiar with Moody's description of NDEs. The three experiencers who reported tunnels in Blackmore's study spontaneously reported the sensation of "darkness"; one experiencer who subsequently agreed to label his sensation tunnel in place of "darkness" might have done so to harmonize his reply with the response suggested by the investigator. In a recent comparison of cases reported before and after 1975 cases, that is, before and after publication of Moody's book Life After Life, NDEs showed an increase in reporting tunnels after 1975 (Athappilly, Greyson, and Stevenson, 2006). In the present study, one experiencer reported the sensation of a cave that, even if considered as a tunnel sensation (I am not suggesting it was), occurred while she was returning back from (not while going to) the other realm, and there was no mention of light at the end of the cave. Furthermore, although a tunnel has been reported in Western studies (Grey, 1985; Ring, 1980; Sabom, 1982), it is not considered to be a core feature of NDEs.

The experience of "beings of Light" was reported by the north Indian experiencers (Pasricha and Stevenson, 1986) but it was not reported by any of the south Indian experiencers. The feature of bright light has been often cited in the Western literature (Eadie, 1992; Moody, 1988; Morse and Perry, 1992; Schoenbeck and Hocutt, 1991), but it was reported in the Indian cases only in the present study. The feature of visibility in the other realm was investigated for the first time.

The feature of positive change in attitude toward death, that is, of reduction in fear of death, and attitude toward life of increased religiosity and generosity was reported in the present series. The features of positive changes in attitude toward life and death, values, and satisfaction with life have been reported in American cases 
(Atwater, 1988; Greyson, 1983, 1994). On the whole, the quality of life in most cases seemed to improve following NDEs.

\section{Conclusion}

The prevalence of NDEs varies within and across cultures. Variations in features also seem to occur across cultures but they do not seem to be true variations for all features. Certain features appear to be culture-specific while others seem common across cultures. The variations in the frequency of occurrence of cases may be due to differences in methodologies adopted by different researchers but that does not seem the only explanation, as I conducted the present study using more or less same survey techniques that I had employed in the previous studies, yet there were variations in the prevalence rate and features. On the other hand, although samples for American and Indian studies were drawn from different populations - hospitals and communities, respectively - similarities in certain features were observed in both cultures. It follows then that some features are perhaps shaped by cultural beliefs, influenced by methodological variation and location of the experiencers at the time of experience, whereas others are universal in content although their presentation may seem to differ.

\section{References}

Athappilly, G. K., Greyson, B., and Stevenson, I. (2006). Do prevailing societal models influence reports of near-death experiences? A comparison of accounts reported before and after 1975. Journal of Nervous and Mental Disease, 194, 218-222.

Atwater, P. M. H. (1988). Coming back to life: The after-effects of near-death experience. New York, NY: Dodd, Mead.

Blackmore, S. J. (1993). Near-death experiences in India: They have tunnels, too. Journal of Near-Death Studies, 11, 205-217.

Director of Census Operations. (1984). Census of India 1981. Series 9: KarnatakaBangalore District (Paper 3 of 1984). New Delhi, India: Office of the Registrar General.

Eadie, B. J., and Taylor, C. (1992). Embraced by the light. Placerville, CA: Gold Leaf Press.

Grey, M. (1985). Return from death. London, England: Arkana.

Greyson, B. (1983). Near-death experiences and personal values. American Journal of Psychiatry, 140, 618-620.

Greyson, B. (1994). Near-death experiences and satisfaction with life. Journal of NearDeath Studies, 13, 103-108.

Hazra, R. C. (1975). Studies in the Puranic records on Hindu rites and customs. Delhi, India: Motilal Banarsidass. (Original work published 1940) 
Kellehear, A., Stevenson, I., Pasricha, S., and Cook, E. (1994). The absence of tunnel sensations in near-death experiences from India. Journal of Near-Death Studies, 13, $109-113$.

Moody, R. A. (1975). Life after life. Covington, GA: Mockingbird Books.

Moody, R. A., and Perry, P. (1988). The light beyond. New York, NY: Bantam.

Morse, M., and Perry, P. (1992). Transformed by the light: The powerful effect of neardeath experiencers on people's lives. New York, NY: Villard.

Pasricha, S. K. (1993). A systematic survey of near-death experiences in South India. Journal of Scientific Exploration, 7, 171-181.

Pasricha, S. K. (1995). Near-death experiences in South India: A systematic survey. Journal of Scientific Exploration, 9, 79-88.

Pasricha, S. K., and Stevenson, I. (1986). Near-death experiences in India: A preliminary report. Journal of Nervous and Mental Disease, 174, 165-170.

Ring, K. (1980). Life at death: A scientific investigation of the near-death experience. New York, NY: Coward, McCann and Geoghegan.

Sabom, M. (1982). Recollections of death: A medical investigation. New York, NY: Harper and Row.

Schoenbeck, S. B., and Hocutt, G. D. (1991). Near-death experiences in patients undergoing cardiopulmonary resuscitation. Journal of Near-Death Studies, 9, 211-218.

Stevenson, I., Pasricha, S., and McClean-Rice, N. (1989). A case of the possession type in India with evidence of paranormal knowledge. Journal of Scientific Exploration, 3, 81-101.

Walker, B. (1968). The Hindu world. New York, NY: Praeger. 